\section{Past and future}

Huw Price

The Arrow of Time. By Peter Coveney and Roger Highfield. Allen: 1990. Pp. 378. £14.95.

Time Journeys. By Paul Halpern. McGraw-Hill: 1990. Pp. 153. Hbk $\$ 19.95, £ 19.95$, pbk \$11.95, £10.95.

THE arrow of time is one of the big unclaimed prizes of modern physics. The problem is to reconcile the temporal asymmetry of thermodynamics with the apparent temporal symmetry of fundamental physical theories. Some major players have wrestled with the issue over the past century or so, but it is still up for grabs and very much in the air of late, having been discussed in recent books by Stephen Hawking ( $A$ Brief History of Time) and Roger Penrose (The Emperor's New Mind), among others.

The Arrow of Time, subtitled "A voyage through science in search of time's greatest mystery", is a lively guide to the current state of play. Its authors are a scientist and a distinguished science journalist. The former, Peter Coveney, is a member of a team that has claimed a novel and fruitful approach to the game (and whose captain, Ilya Prigogine, provides a foreword to the book). The viewpoint is therefore partisan: the authors soon make it clear that they want to argue that the key to the puzzle lies in some of the insights of Prigogine's Brussels school, and in related work in the theory of chaos. This is a bad omen, for as we will see, someone who understands the rules of the game (that is, who grasps the real mystery about temporal asymmetry) should be able to see that the proposed solution is simply addressing the wrong issue. For all its intrinsic interest, it cannot provide an answer to the main question. The result is a bit like Babe Ruth's Guide to Mysteries of Cricket: fine on baseball, but not what we were promised.

The book's basic mistake is to fail properly to distinguish several quite separate issues about time. For a start, the authors overlook an ambiguity in the arrow metaphor, and hence confuse the issue of the directionality of time (that is, the question of whether the Universe is symmetric in time) with the question as to whether time flows. The standard use of the metaphor turns simply on the fact that arrows are effectively one-dimensional objects, with a clear orientation along this single dimension. But some arrows move (unlike, for example, the ones in signposts), and this provides the trap that Coveney and Highfield fall into in saying that time "travels like an arrow" (page 24). Antiquity has given us a perfectly good metaphor for the flow of time, namely the stream or river. The arrow is best kept for simple directionality.

More seriously, the authors also fail to see an important distinction between the issues of directionality and determinism. This emerges early, when they misconstrue Einstein's remark that "the distinction between past, present and future is an illusion". They see this as arising from a "belief in a deterministic world" (page 30;

demolishes one of the main positive suggestions of the book. If chaos shows that the future is open, it also shows that the past is open. It does not yield an arrow of time.

Coveney and Highfield also suggest that the Brussels school's study of nonequilibrium systems provides a key to the resolution of the conflict between thermodynamics and mechanics. It does not. The real puzzle is why there is an arrow of time at all; that is, why the Universe is not simply a thermodynamic equilibrium at all times (except during the inevitable local fluctuations). The theory of nonequilibrium systems may tell us how such systems behave, given that there are some; but it does not explain how they come to be so common in the $\sum$ first place (and all oriented in the same temporal direction). This is "time's greatest mystery", and for all its merits, the theory of nonequilibrium systems does not touch it.

What would touch it would be a cosmological demonstration that the Universe was bound to be in a low-entropy state after the $\mathrm{Big}$ Bang. In their recent books Hawking and Penrose have each discussed proposals of this kind approaches which at one point the present authors dismiss on the odd grounds that the cosmological theories concerned are "less firmly established than the phenomena to be explained" (page 34). Goodbye theoretical conjecture! The cosmological approach is at present inconclusive, but the popular works of some of its advocates Paul Davies, for example, as well as Hawking and Penrose provide the best current lay guides to the search for the arrow of time. Davies - that living testimony to the Universe's ability to create order at a prodigious rate - has also written a recent book

Father Time - still a mystery

see also page 64). In fact the remark is simply an allusion to the familiar notion of a four-dimensional block universe, embodying no objective "present moment" or flow of time. Such a view need not insist that the state of the Universe at one time determines its state at other times.

In general, the issue of determinism is quite distinct from that of the directionality of time. A deterministic system may be asymmetric (for example, in virtue of its boundary conditions) and an indeterministic system may be symmetric. So even if it is true (by no means clear, in my view) that the theory of chaotic evolution "blows apart time-symmetric determinism" (page 206; see also pages 36-37), what it leaves might well be time-symmetric indeterminism. This on chaos and nonequilibrium systems (The Cosmic Blueprint, Simon and Schuster, 1988). To my mind it has the edge on the present work.

Halpern's Time Journeys is subtitled "A Search for Cosmic Destiny and Meaning". We philosophers no longer work this territory, so I will not try to comment on whether the search is successful. The subtitle aside, however, the book is a modest but wide-ranging introduction to ideas about time in science and human culture. It would be useful introductory reading for an undergraduate course on this topic. There are many references to more advanced material.

Huw Price is in the Department of Traditional and Modern Philosophy, University of Sydney, Australia 2006. 